\section{Composition of Silk}

IT is well known that the sericin component of silk is soluble in water, and the fibroin is soluble in certain acids and a variety of salt solutions. The latter can be dialysed to leave a solution of fibroin in water ${ }^{1,2}$, from which water-soluble films can be cast. Ambrose et al. ${ }^{1}$ have shown by infra-red methods that these are in the $\alpha$-configuration, and that, on denaturation by alcohol or ammonium sulphate or by stretching, an incomplete conversion to the insoluble $\beta$-form occurs.

Earlier X-ray studies (Meyer) ${ }^{3}$ showed the crystalline part of the fibroin structure to be made up of extended $\beta$-chains, with side-chains roughly alternately $-\mathrm{H}$ and $-\mathrm{CH}_{3}$, that is, a copolymer repeat of alanine and glycine as in Fig. 1. The larger residues of the polypeptide, such as tyrosine, were associated with the non-crystalline parts of the fibre.<smiles>CCCCC(C)NC(=O)OCC</smiles>

From a survey of published equatorial spacings of $\mathrm{X}$-ray diagrams of silk, it is suggested that this structure may apply only to part of the $\beta$-phase. In this the 'alanine/glycine' component of the crystal structure may be very similar to that of nylon", poly-x-alanine, or polyglycine with reference to the hydrogen-bonding distances. Fig. 2 shows a possible cross-section of the unit cell (or its submultiple) of this phase based on that assigned to polyglycine by Astburys. The second component gives essentially the normal type of photograph of a $\beta$-structure with large side-chains. The $4.65 \mathrm{~A}$. spacing is the 'backbone' hydrogen-bonding distance and that of $\sim 9 \mathrm{~A}$. the side-chain spacing. This structure would be associated with the tyrosine groups, etc., and from chemical evidence it appears likely that these larger groups tend to be associated together in discrete parts of the fibre ${ }^{9}$.

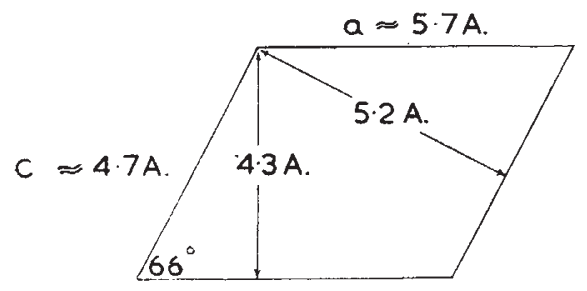

Fig. 2

The latest available analysis gives the serine content of fibroin as about 15 per cent ${ }^{2}$, in contradistinction to 1.5 per cent as used by Meyer and Mark ${ }^{6}$. It is important, therefore, to consider the possible location of these residues in the fibmoin chain with particular reference to their hydrophilic character. Previously, various examples of the isomorphic replacement of groups in fibrous lattices have been found ${ }^{7}$. In particular, Bunn ${ }^{8}$ has demonstrated this in the case of hydrogen and the hydroxyl group in polyvinyl alcohol. It is suggested, therefore, that the $-\mathrm{CH}_{2} \mathrm{OH}$ group of serine may replace $-\mathrm{CH}_{3}$ of alanine in the molecular chain of Fig. 1 , and produce little change in the characteristic monoclinic $\beta$-lattice of the fibroin (Fig. 2). Chemical evidence ${ }^{\theta}$ seems to support the presence of the serine residue in the glycine-alanine parts of the fibroin, although some of the 15 per cent of serine is also contained in the larger residues of the chain, for example, in the orthorhombic $\beta$-lattice. Polyglycine and poly-t-alanine are insoluble in water, but the presence of serine confers solubility on the portion of the chain containing the residues of low molecular weight; thus it is likely that the whole fibroin molecule is in the $\alpha$-form in solution in the sac of the silkworm. Further, the fibroin and sericin are in solution together at approximately 30 per cent concentration ${ }^{10}$, and Imms $^{11}$ describes the production of filament silk as follows : "The silk is secreted in the form of a filament composed of fibroin which is coated with a glutinous substance, sericin, formed by the transformation of the outer layer of the fibroin when the latter is oxidized and combined with water molecules". In the sac, the fibroin and sericin are probably similar polypeptides containing varying proportions of hydrophilic sidechains, with no clear-cut division between the two, but rather a gradation of solubility. The hydrophilic serine and threonine account for 45 per cent of the amino-acids in sericin, and this being more soluble probably retards precipitation of the fibroin.

A possible method of spinning employed by the silkworm would be: (a) alignment of the $\alpha$-chains by extrusion and drawing; (b) extension of the less-soluble $\alpha$-chains, that is, those containing mainly alanine/glycine/serine, into the monoclinic $\beta$-form; (c) partial transformation of other more stable parts of the chains into the orthorhombic $\beta$-form; (d) the retention around the fibroin of the more hydrophilic molecules in solution, forming the sericin fraction of the raw cocoon silk.

This explains the existence of sericin on the surface of the fibres as a consequence of the process of extrusion and coagulation, without the necessity of invoking oxidative processes.

This method of fibre formation differs from those of the rayon industry, where the filament is coagulated from the surface inwards whatever the mode of spinning ; but it explains why such a highly orientated and flexible structure is obtained in these natural filaments. Further, it suggests that earlier regenerated fibroin filaments were brittle and difficult to orientate after coagulation ${ }^{2}$ because sericin is essential to the spinning process as carried out by the silkworm.

A more detailed study of the mechanism is being made. We are indebted to Mr. C. Willoughby, of the Department of Biology of this College, for his assistance.

F. HAPPEY

A. J. HYDE

Department of Textile Industries, Technical College, Bradford.

Jan. 21.

${ }^{1}$ Ambrose, Bamford, Elliott and Hanby, Nature, 167, 264 (1951).

${ }^{2}$ Coleman and Howitt, Proc. Roy. Soc., A, 190, 145 (1947).

s Meyer and Mark, "Natural and Synthetic Polymers", 447.

- Bunn and Garner, Proc. Roy. Soc., A, 189, 39 (1947).

Astbury, Nature, 163, 722 (1949).

- Meyer and Mark, "Natural and Synthetic Polymers", 445.

'Happey, J. Text. Inst., 41, T 381 (1950).

- Bunn, Nature, 161, 929 (1948).

- Drucker and Smith, Nature, 165, 196 (1950).

10 Meyer and Mark, "Natural and Synthetic Polymers", 452.

11 "Textbook of Entomology" (3rd edit., Methuen). 